\title{
EXCESS OF LOSS REINSURANCE AND THE PROBABILITY OF RUIN IN FINITE HORIZON
}

\author{
MARIA De LOURDES CENTENO ${ }^{1}$ \\ ISEG, Technical University of Lisbon
}

July, 1995

\begin{abstract}
The upper bound provided by Lundberg's inequality can be improved for the probability of ruin in finite horizon, as Gerber (1979) has shown. This paper studies this upper bound as a function of the retention limit, for an excess of loss arrangement, and compares it with the probability of ruin.
\end{abstract}

\section{KEYWORDS}

Excess of loss; reinsurance; finite time ruin probability.

\section{INTRODUCTION}

Several studies about the effect of reinsurance on the ultimate probability of ruin (for example Gerber (1979), Waters (1979), Bowers, Gerber, Hickman, Jones and Nesbitt (1987), Centeno (1986) and Hesselager (1990)) have concentrated their attention on the effect of reinsurance on the adjustment coefficient.

Centeno (1986) has used an algorithm suggested by Panjer (1986) to calculate the probability of ultimate ruin, incorporating reinsurance, to show with some examples that the behaviour of this probability and Lundberg's inequality are very similar, both considered as functions of the retention level, provided that the initial reserve is not too small. This is consistent with the figures obtainded more recently by Dickson and Waters (1994) for some other examples and using a different algorithm for the probability of ultimate ruin. In this paper, Dickson and Waters have also calculated finite horizon ruin probabilities, after reinsurance, by adapting the algorithm of De Vylder and Goovaerts (1988) and by an approximation provided by the translated Gamma process. Through an example they show that in continuous time for an excess of loss arrangement, the optimal retention limit in finite horizon can be quite far from the optimum value in infinite horizon. Of course, the sequence of optimal retention levels

\footnotetext{
${ }^{1}$ Research performed under contract $n^{\circ}$ SPES-CT91-0063

ASTIN BULLETIN, Vol 27, No 1, 1997, pp 59-70
} 
converges to the infinite horizon optimal level as the time increases. But, for a finite horizon, Lundberg's inequality can be improved. The purpose of this paper is to show how we can use this improvement to redefine the "optimal" retention limit for an excess of loss arrangement, and to compare this inequality with the ruin probability in finite horizon and continuous time for some examples. Of course, the same methodology can be applied to proportional reinsurance provided that, the moment generating function of the individual claim amounts distribution exists.

\section{ASSUMPTIONS AND PRELIMINARIES}

In the classical risk process, the insurer's surplus at time $t$ is denoted $U(t)$, with

$$
U(t)=u+c t-S(t)
$$

where $u$ is the initial surplus, $c$ is the premium income per unit of time, assumed to be received continuously, and $S(t)$ is the aggregate claims occurred up to time $t .\{S(t)\}_{t \geq 0}$ is assumed to be a compound Poisson process and without loss of generality the Poisson parameter is assumed to be 1 , which means that "time $t$ " is the interval during which $t$ claims are expected. Let $G(x)$ denote the individual claim amount distribution function and again without loss of generality, let us assume that this distribution has mean 1 , which means that the monetary unit chosen is the expected amount of a claim. We further assume that $G(0)=0$, with $0<G(x)<1$ for $x>0$ and also that $G$ is such that its moment generating function exists for $x<T$ for some $0<T \leq \infty$, and that

$$
\lim _{r \rightarrow T} E\left[e^{r X}\right]=\infty \text {. }
$$

We assume that $c$ is greater than 1, i.e. it is greater than the expected aggregate claims in each period. Let $\theta$ be such that $c=1+\theta$.

The ruin probability before time $t$ is

$$
\psi(u, t)=\operatorname{Pr}\{U(s)<0 \text { for some } s, 0<s \leq t\} .
$$

Of course $\psi(u, t)$ is not greater than the ultimate probability of ruin, denoted as $\psi(u)$. Therefore the upper bound given by Lundberg's inequality is valid for finite horizon. Gerber (1979), pp 139, has shown that this bound can be improved in finite horizon. He proved that for $u \geq 0$ and $t>0$

$$
\psi(u, t) \leq \min _{r \geq R}\left\{e^{-r u+t\left[M_{X}(r)-1-r c\right]}\right\},
$$

where $M_{X}(r)$ is the moment generating function of the individual claim amounts and $R$ denotes the adjustment coefficient, defined as the unique positive root of

$$
M_{X}(r)-1=c r \text {. }
$$

In the following we refer to expression (2) as Gerber's inequality. After an integration by parts, inequality (2) can be written as

$$
\psi(u, t) \leq \min _{r \geq R}\left\{e^{-r u+r t\left[\int_{0}^{\infty} e^{r x}(1-G(x)) d x-c\right]}\right\},
$$


and the equation defining the adjustment coefficient as

$$
\int_{0}^{\infty} e^{r x}(1-G(x)) d x=c .
$$

Now suppose that the insurer has an excess of loss arrangement such that when a claim $X$ occurs he is responsible for $\min \{X, M\}$, paying in return per unit of time a reinsurance premium $c(M)$, which we assume to be calculated according to the expected value principle with loading coefficient $\xi$, i.e.

$$
c(M)=(1+\xi) \int_{M}^{\infty}(1-G(x)) d x .
$$

Assuming that the reinsurance premiums are paid continuously, the insurer's surplus at time $t$ is

$$
U(M ; t)=u+(c-c(M)) t-\sum_{k=1}^{N(t)} \min \left\{X_{k}, M\right\},
$$

where $N(t)$ denotes the number of claims up to time $t$. The ruin probability before time $t$ is

$$
\psi(M ; u, t)=\operatorname{Pr}\{U(M, s)<0 \text { for some } s, 0<s \leq t\} .
$$

After this arragement Gerber's inequality becomes

$$
\psi(M ; u, t) \leq \min _{r \geq R(M)}\left\{e^{-r u+r f\left[\int_{0}^{M} e^{r x}(1-G(x)) d x-(c-c(M))\right]}\right\},
$$

where $R(M)$ denotes the adjustment coefficient after reinsurance, i.e. the unique positive root of

$$
\int_{0}^{M} e^{r x}(1-G(x)) d x=c-c(M)
$$

when it exists or zero otherwise. Such a root exists if and only if the expected profit after reinsurance is positive.

We know that the value of $M$ that maximises the adjustment coefficient, when the excess of loss reinsurance premium is calculated according to the expected value principle with $\xi>\theta$, is such that

$$
M=\frac{1}{R} \ln (1+\xi),
$$

(see for example Waters (1979)), minimising then the upper bound provided by Lundberg's inequality.

In the next section we will study the problem that consists in choosing $M$ in such a way that the upper bound provided by (7) is minimised. 


\section{THE PROBLEM AND ITS SOLUTION}

We define as "optimal" retention the value of $M$ that minimises the upper bound of the probability of ruin given by (7). We can write (7) as

$$
\psi(M ; u, t) \leq \exp \left(\min _{r \geq R(M)} f(r, M ; u, t)\right)
$$

where

$$
f(r, M ; u, t)=-r u+r t\left[\int_{0}^{M} e^{r x}(1-G(x)) d x-(c-c(M))\right] .
$$

In the next result we will study the condition under which (11), as a function of $r$, possesses a minimum.

\section{Result 1}

(i) For each $M>0, f(r, M$; $u, t$ ), defined by (11), for $r>0$, has a local minimum and it is unique if and only if the expected surplus at $t$ is positive.

(ii) Suppose that the expected surplus at time $t$ is positive and let $\hat{r}(M)$ be the value of $r$ at which the local minimum of $f(r, M ; u, t)$ occurs. Then $\hat{r}(M) \geq R(M)$, where $R(M)$ is the unique positive root of (8) if it exists or zero otherwise, if and only if

$$
\frac{u}{t} \geq R(M) \int_{0}^{M} x e^{R(M) x}(1-G(x)) d x
$$

\section{Proof:}

(i) It is clear that for $M>0$

$$
\lim _{r \rightarrow 0} f(r, M ; u, t)=0
$$

and, by assumption (1), that also for any $M>0$

$$
\lim _{r \rightarrow \infty} f(r, M ; u, t)=+\infty \text {. }
$$

On the other hand

$$
\frac{\partial f}{\partial r}=-u+t \int_{0}^{M} e^{r x}(1-G(x)) d x-t(c-c(M))+r t \int_{0}^{M} x e^{r x}(1-G(x)) d x
$$

and

$$
\frac{\partial^{2} f}{\partial r^{2}}=2 t \int_{0}^{M} x e^{r x}(1-G(x)) d x+r t \int_{0}^{M} x^{2} e^{r x}(1-G(x)) d x
$$

As (14) is strictly positive for any $M>0$, then $f(r, M ; u, t)$ will have a minimum if and only if the limit of (13) is negative as $r \rightarrow 0$. But

$$
\lim _{r \rightarrow 0} \frac{\partial f}{\partial r}=-u+t\left[\int_{0}^{M}(1-G(x)) d x-(c-c(M))\right],
$$

which is negative if and only if the expected surplus at time $t$ is positive. 
(ii) $\hat{r}(M)$ is the solution of

$$
\frac{\partial f}{\partial r}=0
$$

with $\partial f / \partial r$ given by (13). It is clear that $\hat{r}(M)$ will be greater than or equal to $R(M)$ if and only if $\partial f / \partial r$ is non positive at the point $r=R(M)$, i.e. if and only if condition (12) holds.

Let $M_{0}$ be the minimum of the values for which the expected surplus at time $t$ is non negative, i.e.

$$
M_{0}=\min \left\{M: M \geq 0 \text { and } u+t\left[c-c(M)-\int_{0}^{M}(1-G(x)) d x\right] \geq 0\right\} .
$$

Note that $M_{0}$ will be zero if and only if $u / t \geq \xi-\theta$. Then the following corollary follows from the previous proof.

Corolary 1.1 For each $M>M_{0}$,

$$
\psi(u, t ; M) \leq\left\{\begin{array}{l}
e^{f(\hat{r}(M), u, t, M)} \text { if } \frac{u}{t}>R(M) \int_{0}^{M} x e^{R(M) x}(1-G(x)) d x \\
e^{f(R(M), u, t ; M)} \text { if } \frac{u}{t} \leq R(M) \int_{0}^{M} x e^{R(M) x}(1-G(x)) d x
\end{array}\right.
$$

where $R(M)$ is the unique positive solution of (8) if it exists or zero otherwise and $\hat{r}(M)$ is the unique positive solution of

$$
\int_{0}^{M} e^{r x}(1-G(x)) d x-(c-c(M))+r \int_{0}^{M} x e^{r x}(1-G(x)) d x=\frac{u}{t} .
$$

Hence we can conclude that for some values of $M$ it will be possible to improve the upper bound given by Lundberg's inequality, which implies that in some cases the value of $M$ that minimises the upper bound provided by Gerber's inequality is different from the value of $M$ that maximises the adjustment coefficient. As this maximum is attained at the unique solution of (8) satisfying (9) we can conclude that this value is different from the minimiser of Gerber's inequality if and only if

$$
\frac{u}{t}>R^{*} \int_{0}^{\frac{1}{R^{*}} \ln (1+\xi)} x e^{R^{*} x}(1-G(x)) d x,
$$

where $R^{*}$ is the unique solution of

$$
\int_{0}^{\frac{1}{r} \ln (1+\xi)} e^{r x}(1-G(x)) d x=c-c\left(\frac{1}{r} \ln (1+\xi)\right) .
$$

Let us study the behaviour of Gerber's bound as a function of the retention limit. Notice that 


$$
\begin{aligned}
\min _{M \geq M_{0}} \psi(u, t ; M) & \leq \exp \left(\min _{M \geq M_{0}} \min _{r \geq R(M)} f(r, M ; u, t)\right) \\
& =\exp \left(\min _{r \geq R(M)} \min _{M \geq M_{0}} f(r, M ; u, t)\right)
\end{aligned}
$$

Differentiating $f(r, M ; u, t)$ with respect to $M$ and considering (6) we get

$$
\frac{\partial f}{\partial M}=r t(1-G(M))\left(e^{r M}-(1+\xi)\right)
$$

and differentiating twice

$$
\frac{\partial^{2} f}{\partial M^{2}}=r t\left[r e^{r M}(1-G(M))+\left((1+\xi)-e^{R M}\right) g(M)\right]
$$

which implies that the first derivative is zero if and only if

$$
M=\frac{1}{r} \ln (1+\xi)
$$

and that the second derivative is positive whenever (24) holds. This means that for fixed $r, u$ and $t, f(r, M ; u, t)$ has a local minimum, which is unique and attained at the point $M=\frac{1}{r} \ln (1+\xi)$.

Let $r_{0}=\frac{1}{M_{0}} \ln (1+\xi)$ with $M_{0}$ given by (16). (Note that $r_{0}$ will be finite if and only if $u / t<\xi-\theta$.).

So, minimising $f(r, M ; u, t)$ for $r \geq R(M)$ and $M \geq M_{0}$, is equivalent to minimising $f\left(r, \frac{1}{r} \ln (1+\xi) ; u, t\right)$ for $R^{*} \leq r \leq r_{0}$, where $R^{*}$ is the unique solution to $(20)$.

Differentiating $f\left(r, \frac{1}{r} \ln (1+\xi) ; u, t\right)$ with respect to $r$ we get

$$
\begin{aligned}
\frac{\partial}{\partial r} f\left(r, \frac{1}{r} \ln (1+\xi) ; u, t\right)= & -u+t \int_{0}^{\frac{1}{r} \ln (1+\xi)} e^{r x}(1-G(x)) d x \\
& -t\left(c-c\left(\frac{1}{r} \ln (1+\xi)\right)\right) \\
& +r t \int_{0}^{\frac{1}{r} \ln (1+\xi)} x e^{r x}(1-G(x)) d x
\end{aligned}
$$

and differentiating twice we get 


$$
\begin{aligned}
\frac{\partial^{2}}{\partial r^{2}} f\left(r, \frac{1}{r} \ln (1+\xi) ; u, t\right)= & 2 t \int_{0}^{\frac{1}{r} \ln (1+\xi)} x e^{r x}(1-G(x)) d x \\
& +r t \int_{0}^{\frac{1}{r} \ln (1+\xi)} x^{2} e^{r x}(1-G(x)) d x \\
& -\frac{t}{r^{2}}(\ln (1+\xi))^{2}(1+\xi)\left(1-G\left(\frac{1}{r} \ln (1+\xi)\right)\right) \\
= & t \int_{0}^{\frac{1}{r} \ln (1+\xi)} x^{2} e^{r x} d G(x),
\end{aligned}
$$

which is positive, implying that $f\left(r, \frac{1}{r} \ln (1+\xi) ; u, t\right)$ is a convex function of $r$. That the three terms sum to the right hand side of (26), can be easily checked, by integrating by parts this last expression. Hence we can conclude that there is at most one solution to

$$
\frac{\partial}{\partial r} f\left(r, \frac{1}{r} \ln (1+\xi) ; u, t\right)=0
$$

and that when it exists it is the global minimum of $f\left(r, \frac{1}{r} \ln (1+\xi) ; u, t\right)$.

But on one hand

$$
\lim _{r \rightarrow 0} f\left(r, \frac{1}{r} \ln (1+\xi) ; u, t\right)=0
$$

and

$$
\lim _{r \rightarrow 0} \frac{\partial}{\partial r} f\left(r, \frac{1}{r} \ln (1+\xi) ; u, t\right)=-u-\theta t<0 .
$$

On the other hand, if $u / t<\xi-\theta$, then $r_{0}$ will be finite and

$$
\lim _{r \rightarrow r_{0}} f\left(r, \frac{1}{r} \ln (1+\xi) ; u, t\right)=r_{0} t \int_{0}^{M_{0}}\left(e^{r_{0} x}-1\right)(1-G(x)) d x \geq 0
$$

and if $u / t \geq \xi-\theta$ then

$$
\begin{aligned}
\lim _{r \rightarrow r_{0}} f\left(r, \frac{1}{r} \ln (1+\xi) ; u, t\right) & =\lim _{r \rightarrow r_{\infty}} f\left(r, \frac{1}{r} \ln (1+\xi)\right) \\
& =\lim _{r \rightarrow r_{\infty}}(-r(u-t(\xi-\theta)))=-\infty
\end{aligned}
$$

so we can state the following result.

\section{Result 2}

If $u / t \geq \xi-\theta$ then the upper bound to the ruin probability before time $t$, given by (10), attains its minimum at $M=0$.

If $u / t<\xi-\theta$ then the upper bound, considered as a function of $M$ has an absolute mi-nimum which is attained at the point $M=\frac{1}{r^{*}} \ln (1+\xi)$ with $r^{*}=$ $\max \left(\hat{r}, R^{*}\right)$ where $\hat{r}$ is the solution to 


$$
\int_{0}^{\frac{1}{r} \ln (1+\xi)} e^{r x}(1-G(x)) d x-\left(c-c\left(\frac{1}{r} \ln (1+\xi)\right)\right)+r \int_{0}^{\frac{1}{r} \ln (1+\xi)} x e^{r x}(1-G(x)) d x=\frac{u}{t}(28)
$$

and $R^{*}$ is the unique solution to

$$
\int_{0}^{\frac{1}{r} \ln (1+\xi)} e^{r x}(1-G(x)) d x=(1+\theta)-(1+\xi) \int_{\frac{1}{r} \ln (1+\xi)}^{\infty}(1-G(x)) d x
$$

if such a root exists or zero otherwise.

\section{EXAMPLES}

In this section we give some examples for the problem studied in the previous section and compare the values obtained for the upper bound given by Gerber's inequality with the values of Lundberg's bound and the values of ruin probability in finite horizon.

Example 1: Let us consider first the case of exponential individual claim amounts, i.e. $G(x)=1-\mathrm{e}^{-x}$ for $x>0$. Then the excess of loss reinsurance premium is $c(M)=$ $(1+) \mathrm{e}^{-M}$ and

$$
M_{0}=-\ln \left(\frac{u+t \theta}{t \xi}\right)
$$

Equation (8) defining the adjustment coefficient $R(M)$ is, in this case, equivalent to

$$
\left(1-e^{-(1-r) M}\right) /(1-r)=(1+\theta)-(1+\xi) e^{-M},
$$

and equation (18) defining $\hat{r}(M)$ is equivalent to

$$
\left(\frac{1}{1-r}+\frac{r}{(1-r)^{2}}\right)\left(1-e^{-(1-r) M}\right)-\frac{r}{1-r} M e^{-(1-r) M}-\left[(1+\theta)-(1+\xi) e^{-M}\right]=\frac{u}{t}
$$

$\hat{r}(M)$ will be greater than $R(M)$ if and only if

$$
\frac{u}{t}>\frac{R(M)}{1-R(M)}\left[\frac{1}{1-R(M)}\left(1-e^{-(1-R(M)) M}\right)-M e^{-(1-R(M)) M}\right]
$$

Equations (30) and (31) can be solved for each $M$ by standard numerical techniques given values of $\theta$ and $\xi$.

If $u / t<\xi-\theta$ the upper bound to $\psi(M ; u, t)$ given by (10) is attained at the point

$$
M=\frac{1}{r^{*}} \ln (1+\xi)
$$

with $r^{*}=\max \left(\hat{r}, R^{*}\right)$ where $\hat{r}$ is the solution to equation (31) with $M$ substituted by the right-hand side of (33) and $R^{*}$ is the solution to equation (30) again with $M$ substituted by the right-hand side of $(33)$. 
Let $\theta=0.2$ and $\xi=0.4$. In this case the value of $M$ that minimises the upper bound provided by Lundberg's inequality is $M=1.486$, which gives a value for the adjustment coefficient of $R^{*}=0.226466$. When we minimise the upper bound provided by Gerber's inequality we get a different solution for the excess of loss retention limit if $u / t>0.12075$, the solution being $M=0$ if $u / t \geq 0.2$. Table 1 gives the optimal $M$ for different values of $u / t$.

TABLE 1

'OPTIMAL' RETENTION AS A FUNCTION OF $w / t$, WITH CLAIM AMOUNTS EXPONENTIALLY DISTRIBUTED

\begin{tabular}{cccccccccc}
\hline $\boldsymbol{u} / \boldsymbol{t}$ & 0.125 & 0.13 & 0.14 & 0.15 & 0.16 & 0.17 & 0.18 & 0.19 & 0.2 \\
\hline $\boldsymbol{M}$ & 1.427 & 1.357 & 1.219 & 1.078 & 0.932 & 0.779 & 0.611 & 0.412 & 0 \\
\hline
\end{tabular}

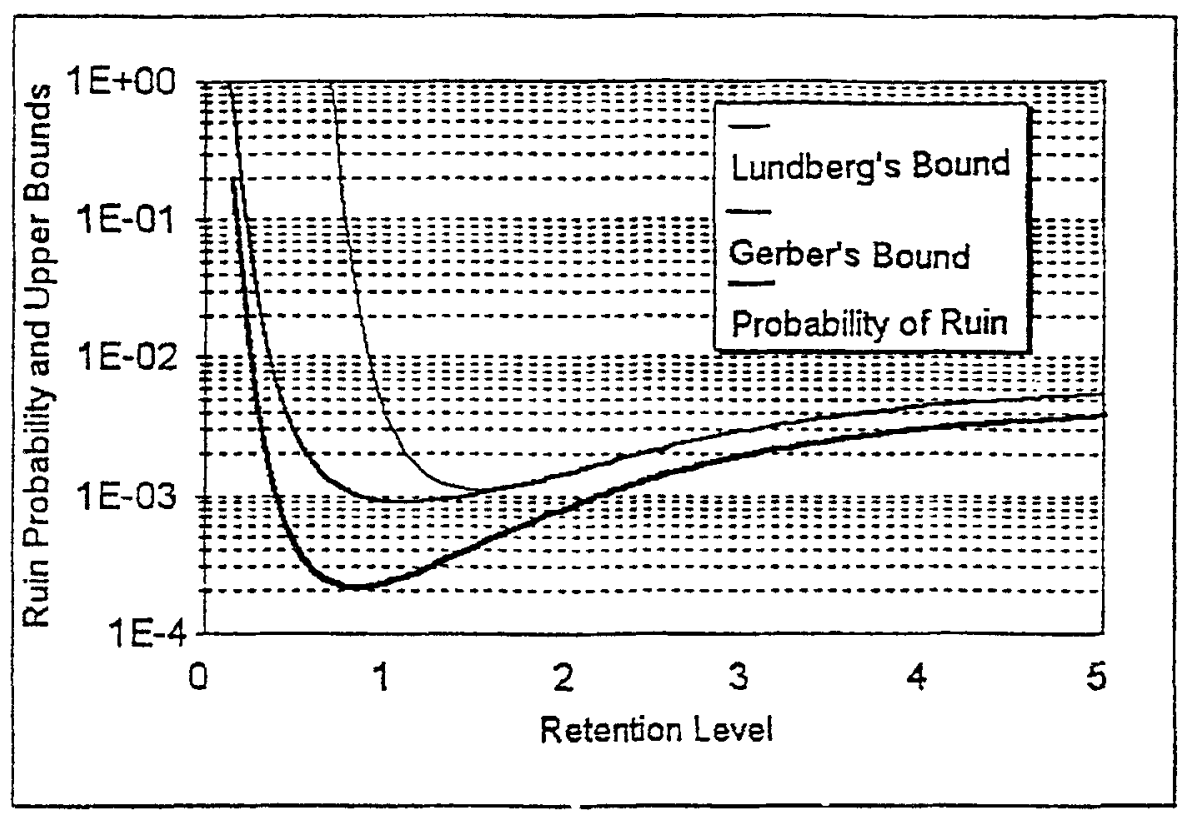

Figure 1: Claim amounts EXPONENTIALly DistribUted

Figure 1 shows calculated values of $\psi(M ; u, t)$, Gerber's upper bound and Lundberg's upper bound for $u=30$ and $t=200$.

Table 2 gives the values attained by these functions at the minimum of each of them (rounded to two decimal places). 
TABLE 2

'OPTIMAL' VALUES WITH CLAIM AMOUNTS EXPONENTIALLY DISTRIBUTED

\begin{tabular}{cccc}
\hline$M$ & $\psi(M ; 30,200)$ & Gerber's bound & Lundberg's bound \\
\hline $\mathbf{0 . 8 3}$ & $0.218 \times 10^{3}$ & $0101 \times 10^{2}$ & $0252 \times 10^{1}$ \\
$\mathbf{1 . 0 8}$ & $0257 \times 10^{3}$ & $0896 \times 10^{3}$ & $0219 \times 10^{2}$ \\
$\mathbf{1 . 4 9}$ & $0442 \times 10^{3}$ & $0104 \times 10^{2}$ & $0112 \times 10^{2}$ \\
\hline
\end{tabular}

The efficiency measure defined by Dickson and Waters (1994) goes from $49 \%$ (= $\psi(0.83 ; 30,200) / \psi(1.49 ; 30,200))$ for the minimiser of Lundberg's bound to $85 \%$ (= $\psi(0.83 ; 30,200) / \psi(1.08 ; 30,200))$ for the minimiser of Gerber's bound.

The probabilities, in all the examples, were calculated using the algorithm of De Vylder and Goovaerts (1988) as re-scaled by Dickson and Waters (1991) and adjusted to take into account reinsurance.

We started by discretizing the individual claim amounts (before reinsurance) on $1 / \beta, 2 / \beta, \ldots$, using the method suggested by De Vylder and Goovaerts (1988). Then, for each value of $M$ we have calculated the net premium (after reinsurance) in the new monetary unit, after which we have calculated the distribution function $F$ of the aggregate claim amounts after reinsurance in a period of time with the rescaled Poisson parameter (in this case - with $t=1$ - the inverse of the net premium). In this way the rescaling parameter depends on the value of the retention. ${ }^{2}$

Then we have used the recursion formula

$$
\begin{gathered}
\hat{\psi}(w, 1)=1-F(w+1), w \leq \bar{w}+(\bar{n}-1), \\
\hat{\psi}(w, n)=1-F(w+1)+\sum_{j=0}^{w+1} f_{j} \hat{\psi}(w+1-j, n-1), w \leq \bar{w}+(\bar{n}-n) ; n=2, \ldots, \bar{n},
\end{gathered}
$$

where $\bar{w}=u \beta$ and $\bar{n}=\{t P\}$ where $P$ denotes the net premium in the new monetary unit and $\{x\}$ denotes the least integer greater than or equal to $x$.

We have used the approximation

$$
\bar{\psi}(w, n) \cong \frac{1}{2}(\hat{\psi}(w-1, n)+\hat{\psi}(w, n))
$$

with $\hat{\psi}(w-1, n)$ to be zero if $w$ is zero, as suggested by De Vylder and Goovaerts (1988), for probabilities in continuous time.

TABLE 3

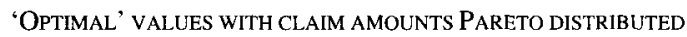

\begin{tabular}{cccc}
\hline \hline $\boldsymbol{M}$ & $\psi(\boldsymbol{M} ; \mathbf{3 0 , 2 0 0 )}$ & Gerber's bound & Lundberg's bound \\
\hline $\mathbf{0 . 8 3}$ & $0102 \times 10^{2}$ & $0549 \times 10^{2}$ & 1000 \\
$\mathbf{1 . 0 3}$ & $0109 \times 10^{2}$ & $0523 \times 10^{2}$ & 0644 \\
$\mathbf{2 . 3 3}$ & $0356 \times 10^{2}$ & $0977 \times 10^{2}$ & 0013 \\
\hline
\end{tabular}

\footnotetext{
${ }^{2}$ Note that with this rescaling we are restricted to evaluate the ruin probabilities for a positive net premium
} 
As $t P$ may be not an integer we have used the following interpolation to calculate the probabilities of the original process

$$
\psi(M ; u, t) \cong \bar{\psi}(u \beta, t P) \cong(\{t P\}-t P) \bar{\psi}(u \beta,\{t P\}-1)+(t P-(\{t P\}-1)) \bar{\psi}(u \beta,\{t P\})
$$

In the calculations of Table 2 we have taken $\beta=100$ and the control parameter, $\varepsilon$, was set at $3 \times 10^{-9}$. This parameter is used for the calculations in the De Vylder and Goovaerts algorithm (see De Vylder and Goovaerts (1988), p. 7). For the calculations of the ruin probabilities necessary to perform Figure 1 we have used $\beta=20$.

Example 2: Consider now the case where $G(x)=1-(1+x)^{-2}$, i.e. individual claims follow a Pareto $(2,1)$ distribution. Let $\theta=0.2$ and $\xi=0.4$ as in the previous example. In this case the equations defining $R(M)$ and $\hat{r}(M)$ require numerical calculations of integrals of the kind

$$
\int_{0}^{M} e^{x}(1-G(x)) d x
$$

Instead of using standard numerical techniques to calculate them, we have calculated $R(M)$ and $\hat{r}(M)$ based on the discretized distribution. Figure 2 shows the ruin probability before time $t=200$, for $u=30$, and both Gerber's and Lundberg's bounds.

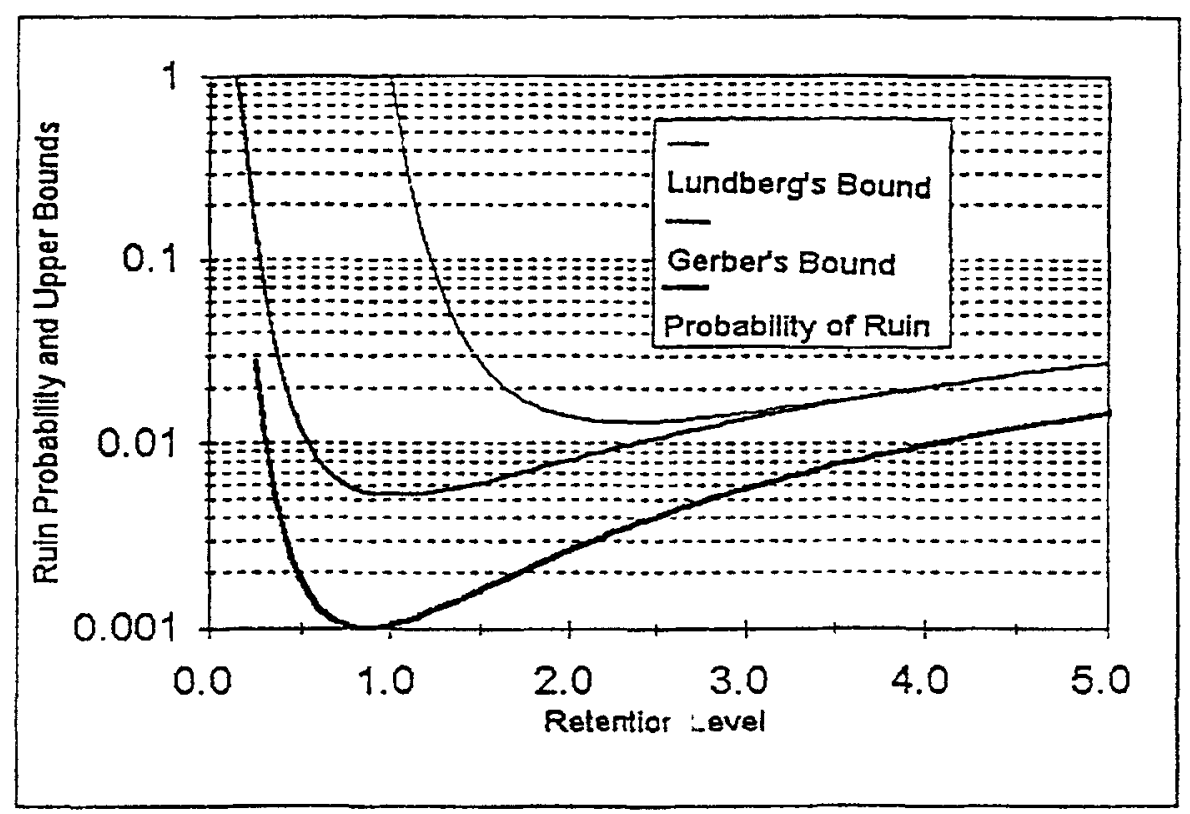

Figure 2: Claim Amounts Pareto distributed

Table 3 equivalent to Table 2, but for the Pareto distribution. The figures are even more indicative. 


\section{CONCLUDING REMARKS}

As we have already mentioned, the optimal retention limit, when the probability of ruin in continuous time with a finite horizon is minimised, can be quite far from the optimal value when the probability of ruin in continuous time with an infinite horizon is considered. However, the calculations of the ruin probabilities in finite horizon are very time consuming, making this criterion less appealing.

Gerber's bound is computationally much easier to deal with than the ruin probability and in the examples considered it provides a solution that is very close to the solution obtained when the probability of ruin is used. The disadvantage of using Gerber's bound is that this bound is not always an improvement on Lundberg's bound - it depends on the value of the ratio of $u$ to $t$. Our advice would be to use Gerber's bound, if it provides an improvement to Lundberg's bound, and use an approximation such as that provided by the translated Gamma process otherwise.

We have shown that when the reinsurance premium calculation principle is the expected value principle, Gerber's bound has a unique minimum. However, this is not true in general. When this is not the case, in all the examples considered, the probability of ruin had a similar behaviour. Some care should be taken in these cases.

\section{REFERENCES}

Bowers, N L , Gerber, H U , Hickman, J C , Jones, D A and Nesbitt, C J (1987) Actuarial Mathematics, Society of Actuaries, Chicago

CENTENo, L (1986) Measuring the effects of reinsurance by the adjustment coefficient, Insurance Mathematics and Economics 5 169-182

DE V YLDER, F AND GoOvaerTs, M J (1988) Recursive calculation of finite-time ruin probabilities, Insurance Mathematics and Economics 7 1-8

DicksON, D AND WATERS, H (1991) Recursive calculation of survival probabilities, Astin Bulletin 21 199221

Dickson, D AND WATERS, H (1994) Reinsurance and ruin, University of Melbourne, CAS, Research Paper Series

GERBER, H.U (1979) An Introduction to Mathematical Risk Theory, S S Huebner Foundation Monographs, University of Pensylvania

Hesselager, O (1990) Some results on optimal reinsurance in terms of the adjustment coefficient, $S_{c a n}$ dinavian Actuarial Journal pp 80-95

PANJER, H H (1986) Direct calculation of ruin probabilities, The Journal of Risk and Insurance 53521 529

W ATERS, H (1979) Excess of loss reinsurance limits, Scandinavian Actuarial Journal pp 37-43 